\title{
Anomalous self-experiences: markers of schizophrenia vulnerability or symptoms of depersonalization disorder? A phenomenological investigation of two cases
}

\author{
Tor Gunnar Værnes ${ }^{\text {ab }}$, Jan Ivar Røssberg ${ }^{c}$, Paul Møller $^{d}$ \\ ${ }^{a}$ Early Intervention in Psychosis Advisory Unit for South-East Norway (TIPS Sør- $\varnothing$ st), Division of Mental Health and \\ Addiction, Oslo University Hospital, Oslo, Norway. \\ ${ }^{\mathrm{b}}$ NORMENT KG Jebsen Center for Psychosis Research, University of Oslo, Norway. \\ 'Division of Psychiatric Treatment Research, Oslo University Hospital and Institute of Clinical Medicine, University of Oslo, \\ Norway \\ ${ }^{\mathrm{d}}$ Dept. of Mental Health Research and Development, Division of Mental Health an Addiction, Vestre Viken Hospital Trust, \\ Norway.
}

\section{Key Words}

Basic self-disturbance - Self-disorders - Anomalous self-experiences - Schizophrenia spectrum disorders - Schizotypal personality disorder - Depersonalization disorder - Clinical high-risk for psychosis

\begin{abstract}
Background: Basic self-disturbance (BSD) is proposed to constitute the clinical core of schizophrenia spectrum disorders, including prodromal states and schizotypy. Anomalous self-experiences (ASEs) are suggested as phenotypic variants of BSD, representing markers of schizophrenia vulnerability. However, ASEs are not restricted to the schizophrenia spectrum, but may also occur in non-psychotic states like depersonalization disorder (DPD). It is unclear to what extent the prevalence and nature of ASEs are differing between the two conditions. The main aim of this paper is to assess and compare ASEs in both conditions, based on literature and two prototypical cases. This might expand the understanding of these phenomena, and strengthen the basis for clinical differentiation.
\end{abstract}

Methods: One patient with schizotypal personality disorder (SPD) and one with DPD were selected from an ongoing clinical high-risk of psychosis study. ASEs were assessed with the Examination of Anomalous Self-Experience (EASE), and analyzed according to the two central dimensions of BSD: diminished self-affection and hyperreflexivity, as well as according to prototypical aspects of depersonalization. The cases were also analyzed and compared with respect to chronology, other symptomatology and psychopathological pathways.

Results: Both cases revealed ASEs reflecting the central dimensions of BSD as well as prototypical aspects of depersonalization. Only the SPD-case however, linked ASEs to psychotic-like ideas of external influence and control. The symptoms had an insidious early childhood onset with no obvious triggers in the SPD-case, and an abrupt adolescence onset triggered by second-time cannabis use and panic anxiety in the DPD-case.

Conclusions: The similarities and differences in ASEs, symptomatology and developmental pathways of the two cases might be accounted for by an updated model of self-disorders. The model proposes that schizophrenia manifests as a result of a combination of early 'primary' onset of ASEs, reflecting disturbances in early neurodevelopment, and later occurring, 'secondary' ASEs of a more defensiveprotective character. In line with this, the DPD-case may be characterized only by secondary ASEs, and thus better protected against psychotic decompensation than the SPD-case, tentatively affected by a combination of primary and secondary ASEs. 
Early Intervention in Psychosis Advisory Unit for South East Norway (TIPS Sør-Øst)

Division of Mental Health and Addiction, Oslo University Hospital (Norway)

Fridtjof Nansens vei 12A, 0369 Oslo

E-Mail: uxvrnt@ous-hf.no

\section{Introduction}

Phenomenologically oriented researchers have proposed that a disturbance in the basic sense of self lies at the core of schizophrenia spectrum disorders (SSDs) [1-5], and also mark the prodrome of these conditions [6, 7]. Sass and Parnas hypothesized that this basic selfdisturbance (BSD) (also termed self-disorder, ipseity disturbance or disturbance of the 'minimal self' $[1,2,8])$ constitute a driving force with respect to the development of positive, negative and disorganized psychotic symptoms [2]. Two intimately connected phenomena characterize BSD. The first is 'diminished self-affection', referring to a diminished sense of basic self-presence, i.e. existing as a vital subject of awareness or agent of action (accordingly also termed 'diminished self-presence' in recent publications $[9,10])$. The second is 'hyperreflexivity', which refers to forms of exaggerated self-consciousness and heightened awareness of normally tacit or implicit aspects of experience. These phenomena are commonly accompanied by a disturbed 'grip' or 'hold' on the cognitive-perceptual world, feelings of existential perplexity, an inadequate grasp of everyday, self-evident meanings ('common sense-disturbances'), and disturbed self-other/self-world boundaries [2, 3]. BSD commonly manifests as a composite of various anomalous self-experiences (ASEs) [2, 11].

The BSD-model is inspired and informed by a large body of classical phenomenologicalpsychiatric literature [12-16], and the model has achieved substantial empirical support during the last two decades. In 2005, a scale (the Examination of Anomalous Self-Experience (EASE)), was published targeting BSD-phenomena [17]. Self-disorders have been found to be specific to schizophrenia compared to other psychotic disorders, and they aggregate in 
schizotypy and prodromal schizophrenia [4, 11, 18-23]. They are also prominent in genetic and clinical high-risk for psychosis conditions, and predict SSDs in non-psychotic and highrisk for psychosis conditions [22-29]. Consequently, ASEs are increasingly considered as strong phenotypic markers of schizophrenia vulnerability, and the identification of ASEs has been suggested as a close-in strategy for identifying subjects with the highest risk of psychosis, particularly with respect to SSDs [25, 30].

However, the specificity of ASEs is not a fully settled matter. ASEs include depersonalization and derealization phenomena, which may also occur outside of the schizophrenia spectrum. These phenomena are commonly described as feelings of unreality and detachment with respect to the self (depersonalization) and the outside world (derealization). Symptoms may occur briefly or prolonged, in the general population, in various mental disorders (e.g. panic disorder or depression), in medical or neurological conditions, or associated with drug use and medication [31]. Longstanding conditions, with these phenomena as the dominant symptoms, are diagnosed as Depersonalization Disorder (DPD) in DSM-IV, grouped among the dissociative disorders [32]. The most typical triggers of chronic depersonalization (DPD) are drug use, severe stress, anxiety disorders and depression [31, 33]. However, in many subjects no immediate triggers are identified. A sudden onset has been found in $40-50 \%$ of the cases, while other cases are more commonly characterized by a gradual, insidious onset $[33,34])$. DPD generally starts in adolescence or young adulthood [31]. A link with experiences of emotional abuse during childhood has been demonstrated, but not with sexual trauma or physical abuse [35].

Looking at recent research and attempts at understanding and conceptualizing DPD, it is evident that the symptomatology of this disorder is not restricted to feelings of unreality and 
detachment. Several studies indicate that the depersonalized condition is characterized by a number of distinct symptoms [33, 34, 36, 37]. These symptoms constitute a clinical composite, termed the depersonalization syndrome, which is suggested to include: 1) feelings of disembodiment (lack of body ownership and/or agency feelings), 2) emotional numbing (disturbed ability to imbue perceived objects or concrete situations with emotional feeling), 3) anomalous subjective recall (a. 'factual' aspect of memory is preserved but unsure if the event really happened, b. disturbances in the capacity for imagery) and 4) derealization (detachment, feeling cut-off from the world, things seem unreal) [38].

According to Schneider and Klosterkötter, alienation phenomena in non-psychotic disorders are different than the depersonalization phenomena found in schizophrenia, the first being characterized by only 'quantitative' reductions in the sense of self, comprising feelings of obscurity, remoteness and unreality $[16,39,40]$. In line with this conceptualization, and referring to Sass and Parnas' BSD-model, Bürgy asserts that disturbances in the sense of mineness of experience constitute a core syndrome in the schizophrenia spectrum not present in DPD [39]. A categorical distinction between DPD and SSDs is reflected in current diagnostic manuals $[32,41,42]$. However, the description of prototypical symptoms in DPD does include several aspects of disturbances in the sense of mineness of experience, as described with respect to feelings of disembodiment, although not including frank first-rank symptoms $[37,38]$. Such similarities seem to question a clear distinction between SSDs and other mental disorders, including DPD, as made by Schneider, Klosterkötter, Bürgy and proponents of the BSD-model [16, 30, 39, 40].

The main aim of this study thus was to explore and compare ASEs in "mild" schizophrenia spectrum conditions, i.e. schizotypy, and in DPD. This is of importance to expand the 
understanding of these phenomena across diagnostic boundaries, and to strengthen the basis for clinical differentiation, particularly with respect to schizophrenia vulnerability. Meaningful relations between symptoms, and the complex relations between symptoms and pathogenic factors, may be best captured and described at an explorative level through case studies [43]. Consequently, we did a phenomenological investigation and comparison of two patients, one with schizotypal personality disorder (SPD) and the other with DPD, who both fulfilled clinical high-risk (CHR) criteria for psychosis.

\section{Methods}

The SPD- and the DPD-case were purposefully selected from an ongoing prospective CHRstudy to highlight the topic of the present paper. The CHR-study takes place at the Early Intervention in Psychosis Advisory Unit for South-Eastern Norway, Oslo University Hospital (TIPS Sør-Øst), and is a part of the TOP-study at the NORMENT K.G. Jebsen Centre for Psychosis Research, Oslo University Hospital. This study has been approved by the Regional Committee for Research Ethics (REK Sør- Øst).

In the CHR-study, the CHR-status was established according to ultra high-risk (UHR) criteria, assessed with the Structured Interview of Prodromal Syndromes (SIPS) [44] and basic symptoms high-risk criteria: the High-Risk criterion Cognitive Disturbances (COGDIS)), outlined in the Schizophrenia Proneness Scale - Adult Version (SPI-A) [45]. Both cases revealed attenuated positive symptoms and deficits in functioning, but they did not completely fulfil UHR-criteria as these symptoms and deficits had not started or worsened the last year. However, both fulfilled COGDIS-criteria, revealing subjectively experienced thought disturbances. 
Diagnoses were established according to DSM-IV [46], including assessment with the Structured Clinical Interview for DSM-IV Axis I disorders (SCID-I). In addition to SPD (assessed as part of the SIPS), the SPD-case was also diagnosed with a remitting depressive disorder and specific phobia (animal type). The DPD-case also fulfilled criteria for panic disorder with agoraphobia in remission, but the enduring depersonalized condition had prevailed in the absence of anxiety symptoms. Her reality testing abilities were intact (fulfilling the DPD-criterion), which is not incompatible with fulfilling CHR-criteria [47, 48].

A thorough semi-structured phenomenological investigation of ASEs was carried out using the EASE. EASE comprises 57 items collected into five domains: 1. Cognition and Stream of Consciousness (17 items), 2. Self-Awareness and Presence (18 items), 3. Bodily Experiences (9 items), 4. Demarcation/Transitivism (5 items), and 5. Existential Reorientation (8 items) [17]. The EASE has been shown to possess very good to excellent internal consistency [22, 49] and interrater reliability [49]. The items were scored according to the continuous 0-4 severity scale, and later converted into and presented as dichotomous scores $(1=$ definitely present, $0=$ absent or questionably present).

Based on these assessments, the two patients were selected as illustrating cases. Due to the phenomenological overlap between symptoms in DPD and SPD, the formal diagnostic differences between the two cases are shortly summarized here. As to SPD-criteria in DSMIV, only the SPD-case fulfilled the general diagnostic criteria for a Personality disorder. He also fulfilled more than the required five specific criteria for SPD, including distortions in cognition, perceptual experiences, affect, behavior and interpersonal functioning (criteria 1, 2, $3,4,5,6,7$ and 8 ). In the DPD-case, the abrupt onset was better explained as symptoms of an Axis I disorder (panic attack, followed by DPD) than a DSM personality disorder. However, 
she displayed symptoms fitting with some of the specific criteria in DSM-IV for SPD (criteria 3 and 6, possibly criterion 2), as well as similar-sounding criteria for schizotypal disorder in ICD-10 [41]. With respect to the DPD-diagnosis, both cases demonstrated prototypical symptoms (criterion A) which were distressing and impacted on functioning (criterion C). However, unlike the DPD-case, these symptoms were better explained as part of another mental disorder (SPD) in the SPD-case (not fulfilling criterion D). Reality testing (criterion B) was also more compromised, although not fully lacking, in the SPD-case [32].

The EASE-interviews were video-taped, viewed several times, and discussed with one of the authors of the EASE (PM). The descriptions of ASEs from the two cases were scrutinized, analyzed and categorized according to the EASE-manual. Based on this first analysis, descriptions of ASEs were again analyzed and this time categorized as examples of one of the two dimensions of BSD: diminished self-affection and hyperreflexivity [2, 30], as well as the four symptom groups constituting the depersonalization syndrome [38]. This second analysis was done on an ad hoc theoretical basis, comparing verbatim statements from the two cases with paradigmatic descriptions of the core dimensions of BSD and the prototypical depersonalization syndrome symptom groups $[2,38,50]$. The descriptions and categorization of ASEs in the two cases were then compared with each other, item by item. Verbatim statements were selected to illustrate similarities and differences between the two cases. A few additional statements from the SIPS-interviews were also included.

The cases were also analyzed and compared with respect to chronology, other symptoms and psychopathological pathways. Information regarding background and medical history was collected from medical journals and through semi-structured interviews with the patients. The accounts of the background, medical history, life situation and symptoms of the two cases 
were anonymized. Both patients signed a written consent approving the publishing of these accounts in a peer-reviewed scientific paper. All interviews and primary analyses of the interviews were undertaken by the first author who had undergone courses and scientific training with respect to the assessment instruments. Diagnoses and CHR-status were systematically discussed with JIR and PM, two experienced psychiatrists and researchers. The EASE scores were discussed in detail with PM, one of the authors of the EASE scale.

\section{Results}

The SPD-case

Robert is a 21-year-old student in information technology, who lives by himself in a student house. The last couple of years he had been lagging behind with his studies, attributing this to disturbances in thinking and attention. Further, he described marked feelings of unreality and detachment, and he was thinking that possibly some external force influenced and tormented him. He appeared attentive and organized in his speech during the interview, although contained. His demeanor seemed restricted, emotionally detached and a bit wary, but not unfriendly.

Feelings of unreality and detachment had been present since early childhood, with no obvious triggers, accompanied by marked feelings of distrust of others, and existential ruminations about the existence of others/the external world. His parents divorced when he was three years old, and he lived primarily with his mother during childhood. He described his mother as kind and caring. His father was described as kind as well, but relations with him became distanced during adolescence. No marked childhood adversities were described, but he had few friends and he disliked school. Some attention and memory difficulties had been present through childhood, but he managed to get average to good degrees in school. During and following a difficult relationship with his first girlfriend at the age of 15-16, he developed more psychoticlike solipsistic ideas, as if he was the only real person alive, as well as ideas of external influence/control, although he was not fully convinced. Concurrently, his cognitive disturbances became more marked and depressive symptoms emerged. He had no history of drug abuse, but presently his consumption of alcohol was quite high and regular. He received short-term psychotherapy for depression and relational problems at the age of sixteen and twenty, with partial effect. At the age of twenty-one, he revealed his psychotic-like ideas for the first time to a psychiatrist. After assessments at a specialized outpatient clinic he was not considered psychotic, but was referred to the present study on CHR-conditions. Before and during baseline assessments in the CHR-study, he was treated with the antipsychotic quetiapin, $50 \mathrm{mg}$ daily, a dose far below the lowest antipsychotic level.

\section{The DPD-case}

Eva is an 18-year-old high school student who presented with severe and persistent symptoms of depersonalization and derealization, as well as fluctuating, remitting symptoms of anxiety, and visual and auditive perceptual disturbances (not hallucinations). Alongside these symptoms, she had been questioning the reality of self/others/world. She was attentive and engaged in the conversation with the interviewer, but her emotional and facial expressivity 
was decreased. Although reporting disturbances in thinking, this was not reflected in her speech.

Eva's parents divorced when she was seven years old. After this incidence, she lived with her mother and two younger siblings. During childhood and adolescence, she often experienced her mother as moody, emotionally abusive or neglectful. Her father was diagnosed with schizophrenia when she was a child. She visited him almost weekly, but experienced his irregular and odd behavior as a continuous burden. In spite of these adversities, she excelled at school and in sport activities, had many friends, and functioned well socially during childhood.

Present symptoms had a sudden onset three years ago, during an episode of second time cannabis use in which she experienced a first-time panic attack. In the year preceding this episode, the relationship with her mother was more marked by arguments and conflict, she had been involved in some mild delinquent behavior, and she was more devoted to religious and metaphysical ideas than before. She had also been afflicted by migraines and cluster headaches during adolescence. Following the episode, she was referred to a Child and Adolescent outpatient unit, where she was diagnosed with an unspecified anxiety disorder. She was treated the next $2 \frac{1 / 2}{2}$ years with psychotherapy and medication (the antidepressant sertraline 50-100 mg daily, $28 \mathrm{mths}$, the antipsychotic risperidone $1-1.5 \mathrm{mg}$ daily (subantipsychotic dose), $22 \mathrm{mths}$ ). During the first year of treatment, symptoms remained strong, including global anxiety and several panic attacks, and she was not able to attend school. Then her symptoms gradually remitted and she went back to school, although a stable depersonalized and emotionally numb state prevailed. She has never used cannabis or other illegal drugs again, and has a low and sporadic consumption of alcohol. Symptoms recurred and increased 4-5 months after discharge, and she fell out of school once more. Then she was referred to a specialized outpatient clinic, where she again was treated with sertraline, $25 \mathrm{mg}$ daily. Shortly after, she was referred to the present CHR-study, due to her feelings of unreality and perceptual disturbances.

As can be seen, the two cases differed significantly with respect to medical history, childhood adversities and childhood functioning. In the SPD-case, ASEs had been present since early childhood, with no obvious triggers, while ASEs and anxiety symptoms commenced more abruptly in mid-adolescence in the DPD-case, triggered by an episode of cannabis use. ASEs had a stable "all day long"-character in both cases, but in mid-adolescence gave rise to enduring psychotic-like symptoms, i.e. ideas about external influence/control in the SPD-case, unlike the DPD-case. While both cases reported longstanding emotional numbness after symptom onset, the DPD-case reported more anxiety symptoms while the SPD-case reported more depressive symptoms. In contrast to the SPD-case, the DPD-case reported emotional abuse and neglect during childhood and adolescence, and, surprisingly, had a family history 
of schizophrenia. From early childhood on, the SPD-case was described as withdrawn, emotionally detached and distrustful with other people, with attention and memory difficulties, while the DPD-case was described as high functioning socially and in school.

Tables 1 and 2 present illustrating quotes on ASEs reflecting the two core dimensions of the BSD-model (diminished self-affection and hyperreflexivity), the corresponding EASE-items, and the EASE-scores. The two cases presented many examples of EASE-items from Domain 1 Cognition and Stream of Consciousness and Domain 2 Self-Awareness and Presence, reflecting the two BSD-core dimensions in both. However, the cases also differed with respect to which EASE-items were present, and how they were presented. Unlike the DPD-case, the SPD-case displayed disturbances in self-, time- and world-experience that seemed more entrenched and characterized by a more severe 'externalization' (in this context meaning that aspects of the self, like thoughts or actions, seem to be influenced by or originate from some external, alien force [51]), manifesting as a variety of paranoid, grandiose and solipsistic "first-rank-like" ideas. Several of the quotes from the SPD-case in table 1, 2 and 3 illustrate these kinds of disturbances and attributions. Reflecting this attitude, the SPD-case presented more items from domain 5 Existential Reorientation and only the SPD-case presented an item in domain 4 Demarcation/Transitivism.

In contrast with the SPD-case, the DPD-case described experiential anomalies marked by a strong feeling of a sudden change in self-experience ('My whole state of mind has changed completely, it's indescribably strange', Table 2). This change was accompanied by an allencompassing perplexity, exaggerated self-consciousness and hyperreflexivity, but not to attributions to external forces. Although the SPD-case also described anomalies in selfexperience, these were not as extensively and spontaneously described as in the DPD-case. 
They seemed more focused on disturbances in cognition and his indifferent mood. Only the DPD-case endorsed EASE-items from Domain 3 Bodily Experiences, possibly reflecting this difference between the two cases.

Insert Table 1 and 2 here

Table 3 illustrates experiences reflecting the depersonalization syndrome as well as corresponding EASE-items. Both participants experienced symptoms from all the depersonalization symptom groups: feelings of disembodiment, emotional numbing, anomalous subjective recall, and derealization.

Insert Table 3 here

\section{Discussion}

The two core dimensions of the BSD-model, as well as prototypical aspects of the depersonalization syndrome, were represented in both the SPD- and the DPD-case. The two cases differed, however, with respect to the tendency for 'externalization' and delusional-like elaboration (stronger in SPD), as well as experienced perplexity and change in self-experience (stronger in DPD). Additionally, they differed with respect to timing and mode of onset, childhood adversities, family history of mental disorders, and childhood social and cognitive functioning.

Phenomenological aspects and differential diagnostics

The presence of the core dimensions of BSD in both cases, including disturbances in the sense of mineness of experience, challenges the view that such disturbances are restricted to the schizophrenia spectrum, as asserted by Bürgy [39]. However, these results are in line with 
both historical and contemporary phenomenological analysis of chronic depersonalization and DPD. These studies describe these kinds of disturbances among the characteristic features of the depersonalization syndrome (using terms like 'feelings of loss of agency' and 'lack of body ownership feelings') [36-38, 52].

With respect to the DPD-case, these results are to a high degree in congruence with a recent study by Sass et al [53]. This study examined the similarities between the descriptions of items in the EASE-manual with those described in published accounts of severe depersonalization. In these accounts, examples of a match with the majority of EASE-items were found (41 of the 57 items), including items mapping a diminishment in the sense of ownership and agency. The authors stated that depersonalization "represents the clearest nonschizophrenic (and non-psychotic) manifestation of diminished self-affection" ( $\mathrm{p}$ 432). Like our study (see quotes from the DPD-case in Table 1 and 2), these accounts also involved clear manifestations of hyperreflexivity. Even the quotes in Table 3, illustrating aspects of the depersonalization syndrome, match the description of EASE-items reflecting at least one of these dimensions: diminished self-affection. Thus in both studies, complementarity between these BSD-dimensions in non-schizophrenic conditions was demonstrated [53]. Given the overlap with the BSD-dimensions, and the fact that depersonalization and derealization syndrome is mentioned among the criteria for schizotypal disorder in the diagnostic manual ICD-10 [41], it is not unexpected that also the SPD-case presented with symptoms fitting with prototypical aspects of the depersonalization syndrome.

The empirical justification of the diagnosis of DPD has been criticized [54], and it could be argued that the presence of BSD-phenomena in chronic depersonalized states are to be expected, because patients diagnosed with DPD may in fact belong to the schizophrenia 
spectrum. However, the distinctiveness of DPD as a disorder with consistent clinical features (the symptoms constituting the depersonalization syndrome), which rarely develops into schizophrenia or other spectrum disorders, has been reinforced in recent years through large case-studies and factor analysis, although more studies are still needed [33, 34, 36, 37, 55].

Still, the striking similarities between our two cases on a phenomenological level seem to question the clear categorical separation of non-psychotic disorders like DPD from schizophrenia spectrum disorders, like SPDs. It is widely recognized and documented that mental disorders, symptoms, pathogenic factors and psychopathological processes overlap, blend into each other and are characterized by continuous dimensions [56-58]. In accordance with such a dimensional view, it has been suggested that depersonalization phenomena and ASEs may manifest in various degrees of severity on the same continuum, from the transient phenomena in the general population, via moderate variants in several non-psychotic conditions, to the most severe and stable forms found in schizophrenia [53].

The description of symptoms in our two cases, as well as the results from the Sass et al study, seems to fit well with this continuum model. We did not find clear examples in the DPD-case of EASE-items exhibiting what may be the most profound distortions of basic self-awareness, social identity and self-world relationships, e.g. Sense of change in chronological age (2.10), Confusion with the other (4.1) or with own specular image (4.2), Feelings of centrality (5.2) and Solipsistic grandiosity (5.7) (although she did entertain solipsistic feelings and thoughts about possibly being the only "real" person alive, related to the experience that other people looked 'mechanical'). These are items possibly more pathognomonic for SSD conditions [53], and several of them were also present in our SPD-case (2.10, see Table 1, 5.2, 5.7). 
Fuchs has presented a dimensional model of transition from basic and prodromal selfdisorders and depersonalization phenomena to prototypical ego disorders (involving Schneider's first-rank symptoms) in schizophrenia, which may further illuminate both the affinities and the discrepancies between the two cases. The model involves four progressive stages: 1. Alienation of operative intentionality, resulting from a lack of mineness or ipseity, 2. Disintegration of the intentional arc, a rendering-explicit and objectification of single elements, 3. Externalization (attribution to external force), experienced in an 'as if' mode, and 4. Breakdown of the 'as if' and transition to delusion [51]. Looking at the verbatim statements from the SPD-case, he seems closer to this "breakdown of the 'as if" than the DPD-case, considering the more severe externalization of symptoms (stage 3 ) and cognitive attributions of ASEs to external forces (stage 3 and 4). The DPD-case present with many examples of stage 1 and 2, and even some examples fitting with the description of stage 3, but the progression of symptoms seems to have halted at this stage.

We did also find some discrepancies between the findings in our study and the Sass et al study [53]. Our DPD-case endorsed some EASE-items assessed as not present in the study by Sass et al. This included items from Domain 1: Thought interference (1.1) ('I can't control it, I don't want to think about it, it pops up'), Silent thought echo (1.5) ('Something is played on and on again in my mind') and Disorder of short-term memory (1.13) ('My memory is miserable, people notice it'), and Domain 5: Magical ideas (5.3) (see Table 1, last quote). Possibly, this could be related to the fact that these items were specifically asked for during the EASE-interview, contrary to the depersonalization accounts in the Sass et al study which did not involve use of the EASE. However, in light of a dimensional model, we may possibly posit our DPD-case closer to a psychosis/schizophrenia pole than most DPD-cases, considering her family history of schizophrenia and the cognitive disturbances reflected in the 
presence of EASE-items from Domain 1, including symptoms constituting the COGDIScriteria.

Psychopathological pathways - primary and secondary BSD

The similarities and differences between the two cases with respect to phenomenological aspects may reflect similarities and differences in pathogenic factors and psychopathological processes. A recently updated model of self-disorders in schizophrenia, proposed by Sass and Borda, may be enlightening in this respect $[9,10]$. They suggest that self-disorders are partly constituted by primary and partly by secondary factors or variants of BSD. Primary factors are hypothesized to reflect early childhood disturbances in neurodevelopment, which involve disruptions in sensorimotor functions and intermodal perceptual integration, including disturbances in somatosensory feedback $[9,59,60]$. Somatosensory feedback is assumed to be indispensable for the normal experience of feelings, existence, consciousness and the self [61]. These disruptions may manifest in an automatic and pre-reflective form as a diminished feeling of unity and/or ownership with respect to the body and mental life (i.e. as primary diminished self-presence/self-affection, operative (primary) hyperreflexivity, and primary disturbed 'grip' of the world), and may impede the ability to apprehend the world in a holistic and vital fashion $[9,59]$. It is possible that the early insidious onset in the SPD-case reflect such primary forms of BSD, which may further have manifested as disturbances in social and cognitive functioning, as well as in personality development.

Secondary factors too, are proposed to represent core dimensions of BSD (secondary forms of diminished self-presence/self-affection, (reflective) hyperreflexivity, and disturbed 'grip' of the world), but these are considered as a kind of defensive-compensatory reactions to primary factors and other stressors, e.g. interpersonal strain, demands or trauma. These secondary factors may occur early in the life-course too, but they are likely to get more pronounced 
during adolescence and early adulthood, probably affected by the rapid neurocognitive development of the prefrontal lobes in the adolescence [10]. Secondary factors may also be associated with disturbances in the activation of the Default Mode Network (DMN). Psychologically, these DMN-disturbances may imply withdrawal from the external world, and concomitant hyperreflexive awareness of "inner" experiences [10, 62].

According to Sass and Borda, ASEs in DPD correspond closely to those in schizophrenia, but are only secondary and defensive in nature. Childhood and adolescent adversities and trauma are common both in schizophrenia and in SPD, and may contribute to secondary ASEs/forms of BSD, both in SSD and in DPD [63-65]. This model is intriguing because it offers a transdiagnostic perspective on the psychopathological role of internal and external stressors in generating ASEs. The abrupt onset of ASEs in our DPD-case may have a secondary nature possibly related to both present stressors (the cannabis episode as well as prevailing conflicts in the family?) and childhood adversities (emotional neglect and abuse, in line with other studies on childhood trauma in DPD [35]).

The differences in the type and timing of onset of ASEs may additionally be an explanation for the difference between the two cases with respect to experiences of perplexity. In the DPD-case, a sudden, extreme transformation of her experiential world took place three years ago. The sudden, 'de novo' mode of onset triggered by cannabis, commonly associated with a terrifying, 'bad-trip' experience, is a clinical pattern repeatedly found in DPD [33, 34, 66-68]. The onset of symptoms in the DPD-case was followed by strong, enduring feelings of unreality and perplexity marking her depersonalized state. In the SPD-case however, the insidious childhood onset and his long-prevailing depersonalized state turned the strangeness 
of the world into the new "normality" of his childhood and adolescence life. Hence, the experiences of perplexity probably became less pronounced even though still present [62].

Interestingly, in parallel with the suggested neurobiological substrate of BSD in schizophrenia [59], the neurobiology of depersonalization and derealization also seem to be characterized by disturbances in perceptual integration. These disturbances are proposed to involve hyperactive prefrontal inhibitory mechanisms suppressing activity in emotional-related areas like the amygdala, insula and other limbic-related structures. These inhibitory processes result in reduced autonomic response to emotionally salient stimuli, reduced somatosensory feedback and deficiencies in interoception. This probably manifest as emotional numbness and qualitative changes in the experience of self, including unreality and detachment feelings [6971]. Disturbances in multisensory integration in DPD may also involve certain parietal networks, considered important for feelings of embodiment, body ownership and agency [38].

Although disruptions in perceptual integration may explain feelings of unreality and detachment in both SSDs and DPD, some of the psychopathological pathways and underlying neurobiological processes may still differ. While these disruptions may reflect primary disturbances in early neurodevelopment in schizophrenia, they are suggested as neurobiological responses for dealing with stress and anxiety (“defensive" processes) in DPD [70], usually occurring at a later stage in life. Accordingly, disruptions in multisensory integration in schizophrenia may be more severe and encompassing than in DPD, involving early structural alterations which we may not find in DPD. Although DPD-patients often present with subtle subjective impairments in short-term-memory, recall, imagery and attention $[72,73]$, they do not in general demonstrate the more severe cognitive disturbances centering on cingulate and prefrontal functioning more typical for schizophrenia [74, 75]. 
According to Sass and Borda [10], secondary factors constitute a "second hit" important for the development of full-blown schizophrenia. Primary factors are suggested to have adverse effects on the formation of a core or basic self, rendering this fragile self as particularly vulnerable when 'hit' by secondary factors. In prodromal states, this may involve a progressive psychopathological process finally ending in psychotic "first-rank" symptoms $[16,51]$. Looking at our DPD-case, the investigation of childhood functioning did not reveal any signs of being compromised by primary BSD. Thus, she may have been well protected with respect to a psychotic decompensation after the onset of ASEs. Possibly, the increased prefrontal inhibitory activity considered characteristic for depersonalized conditions, may have acted as a protective buffer as well. Still, her family history of schizophrenia, as well as the presence of cognitive disturbances (reflected in fulfilling COGDIS-criteria), may indicate that she could be at a higher risk of psychosis than the majority of DPD-patients.

Considering the SPD-case, primary factors may have been prominent, but a combination of primary and secondary factors may still lie behind the present clinical picture. He describes a worsening in his symptoms around the age of 15-16, which could be the effect of secondary factors deriving from primary factors (e.g. manifesting as prototypical schizotypal trait disturbances in interpersonal functioning), combined with life stressors like a troublesome relationship with his first girlfriend. However, a full psychotic decompensation has not followed, possibly due also in his case to neurocognitive protective mechanisms. It has been found that many SPD patients do not display the frontal lobe reductions observed in schizophrenia, possibly protecting them from full-blown psychosis [76]. 


\section{Conclusions}

This phenomenological investigation demonstrates striking similarities in the presence of ASEs between a SPD-case and a DPD-case, reflecting the two core BSD-dimensions and the four prototypical aspects of the depersonalization syndrome. Differences between the two, particularly with respect to the mode of onset and cognitive elaborations/attributions of ASEs, may reflect the predominance of primary (most prominent in the SPD-case) versus secondary forms of BSD (most prominent in the DPD-case). Closely considering psychopathology, social and medical history in light of this updated BSD-model may enhance the specificity of ASEs as phenotypic markers of vulnerability for SSDs and psychosis. Even though experiencing what appears to be many similar kinds of ASEs, DPD-patients may normally be better protected against psychosis, as their neurodevelopment might be less or not compromised by early dysregulation/primary factors.

This is to our knowledge the first empirical case study comparing assessments of ASEs based on EASE-interviews in a DPD-patient and a SPD-patient. The phenomenological investigation of two cases allows thorough, detailed and diverse scrutiny, but evidently limits the generalizability. Although the DPD-case presented with a typical clinical picture with respect to symptoms and medical history, she is still possibly somewhat atypical considering her family history of schizophrenia and cognitive disturbances, with repercussions for the generalizability of the results. However, considering a dimensional perspective on BSD and depersonalization, she is an interesting and illustrating case. This study creates a rationale for new hypotheses and further research in this field, which should recruit larger samples of nonpsychotic depersonalized conditions, and compare with SSD- or CHR-samples, based on the use of EASE and other appropriate measures.

References 
1 Nelson B, Parnas J, Sass LA: Disturbance of minimal self (ipseity) in schizophrenia: clarification and current status. Schizophr Bull 2014;40:479-482.

2 Sass LA, Parnas J: Schizophrenia, consciousness, and the self. SchizophrBull 2003;29:427-444.

3 Sass LA: Self-disturbance and schizophrenia: structure, specificity, pathogenesis (Current issues, New directions). Schizophr Res 2014;152:5-11.

4 Handest P, Parnas J: Clinical characteristics of first-admitted patients with ICD-10 schizotypal disorder. BrJPsychiatry Suppl 2005;187:s49-s54.

$5 \quad$ Parnas J: Self and schizophrenia: A phenomenological perspective; The self in neuroscience and psychiatry. New York, NY, Cambridge University Press; US, 2003, pp 217241.

6 Parnas J, Jansson L, Sass LA, Handest P: Self-experience in the prodromal phases of schizophrenia: A pilot study of first-admissions. Neurology Psychiatry and Brain Research 1998;6:97-106.

$7 \quad$ Moller P, Husby R: The initial prodrome in schizophrenia: searching for naturalistic core dimensions of experience and behavior. SchizophrBull 2000;26:217-232.

8 Parnas J: Self and schizophrenia: A phenomenological perspective. [References], 2003, pp 217-241.

9 Borda JP, Sass LA: Phenomenology and neurobiology of self disorder in schizophrenia: Primary factors. Schizophr Res 2015;169:464-473.

10 Sass LA, Borda JP: Phenomenology and neurobiology of self disorder in schizophrenia: Secondary factors. Schizophr Res 2015;169:474-482.

11 Parnas J, Handest P, Jansson L, Saebye D: Anomalous subjective experience among first-admitted schizophrenia spectrum patients: empirical investigation. Psychopathology 2005;38:259-267.

12 Minkowski E: La Schizophrènie. Psychopathologie des Schizoides et des

Schizophrènes., Paris, France: Payot, 1927.

13 Blankenburg W, Mishara AT: First steps toward a psychopathology of "common sense". Philosophy, Psychiatry, \& Psychology 2001;8:303-315.

14 Huber G, Gross G: The concept of basic symptoms in schizophrenic and schizoaffective psychoses. Recenti ProgMed 1989;80:646-652.

15 Gross G: The 'basic' symptoms of schizophrenia. BrJPsychiatry Suppl 1989:21-25.

16 Klosterkotter J: The meaning of basic symptoms for the genesis of the schizophrenic nuclear syndrome. Jpn J Psychiatry Neurol 1992;46:609-630.

17 Parnas J, Moller P, Kircher T, Thalbitzer J, Jansson L, Handest P, Zahavi D: EASE: Examination of Anomalous Self-Experience. Psychopathology 2005;38:236-258.

18 Parnas J, Handest P, Saebye D, Jansson L: Anomalies of subjective experience in schizophrenia and psychotic bipolar illness. Acta PsychiatrScand 2003;108:126-133.

19 Nordgaard J, Parnas J: Self-disorders and the schizophrenia spectrum: a study of 100 first hospital admissions. Schizophr Bull 2014;40:1300-1307.

20 Haug E, Lien L, Raballo A, Bratlien U, Oie M, Andreassen OA, Melle I, Moller P: Selective aggregation of self-disorders in first-treatment DSM-IV schizophrenia spectrum disorders. J Nerv Ment Dis 2012;200:632-636.

21 Nelson B, Thompson A, Yung AR: Not all first-episode psychosis is the same: preliminary evidence of greater basic self-disturbance in schizophrenia spectrum cases. Early Interv Psychiatry 2013;7:200-204.

22 Raballo A, Parnas J: The Silent Side of the Spectrum: Schizotypy and the Schizotaxic Self. SchizophrBull 2010 
23 Raballo A, Saebye D, Parnas J: Looking at the Schizophrenia Spectrum Through the Prism of Self-disorders: An Empirical Study. SchizophrBull 2009

24 Davidsen KA: Anomalous self-experience in adolescents at risk of psychosis. Clinical and conceptual elucidation. Psychopathology 2009;42:361-369.

25 Nelson B, Thompson A, Yung AR: Basic Self-Disturbance Predicts Psychosis Onset in the Ultra High Risk for Psychosis "Prodromal" Population. Schizophr Bull;2012:Feb.

26 Parnas J, Raballo A, Handest P, Jansson L, Vollmer-Larsen A, Saebye D: Selfexperience in the early phases of schizophrenia: 5-year follow-up of the Copenhagen Prodromal Study. World Psychiatry 2011;10:200-204.

27 Koren D, Reznik N, Adres M, Scheyer R, Apter A, Steinberg T, Parnas J:

Disturbances of basic self and prodromal symptoms among non-psychotic help-seeking adolescents. Psychol Med 2013;43:1365-1376.

28 Raballo A, Pappagallo E, Dell' Erba A, Lo Cascio N, Patane M, Gebhardt E, Boldrini T, Terzariol L, Angelone M, Trisolini A, Girardi P, Fiori Nastro P: Self-Disorders and Clinical High Risk for Psychosis: An Empirical Study in Help-Seeking Youth Attending Community Mental Health Facilities. Schizophr Bull 2016;42:926-932.

29 Klosterkotter J, Hellmich M, Steinmeyer EM, Schultze-Lutter F: Diagnosing schizophrenia in the initial prodromal phase. ArchGenPsychiatry 2001;58:158-164.

30 Nelson B, Raballo A: Basic Self-Disturbance in the Schizophrenia Spectrum: Taking Stock and Moving Forward. Psychopathology 2015;48:301-309.

31 Reutens S, Nielsen O, Sachdev P: Depersonalization disorder. Current opinion in psychiatry 2010;23:278-283.

32 Diagnostic and statistical manual of mental disorders: DSM-IV-TR. Washington, DC, American Psychiatric Association, 2000.

33 Simeon D, Knutelska M, Nelson D, Guralnik O: Feeling unreal: a depersonalization disorder update of 117 cases. J Clin Psychiatry 2003;64:990-997.

34 Baker D, Hunter E, Lawrence E, Medford N, Patel M, Senior C, Sierra M, Lambert MV, Phillips ML, David AS: Depersonalisation disorder: clinical features of 204 cases. Br J Psychiatry 2003;182:428-433.

35 Simeon D, Guralnik O, Schmeidler J, Sirof B, Knutelska M: The role of childhood interpersonal trauma in depersonalization disorder. Am J Psychiatry 2001;158:1027-1033.

36 Simeon D, Kozin DS, Segal K, Lerch B, Dujour R, Giesbrecht T: De-constructing depersonalization: further evidence for symptom clusters. Psychiatry Res 2008;157:303-306.

37 Sierra M, Baker D, Medford N, David AS: Unpacking the depersonalization syndrome: an exploratory factor analysis on the Cambridge Depersonalization Scale. Psychol Med 2005;35:1523-1532.

38 Sierra M, David AS: Depersonalization: a selective impairment of self-awareness. Conscious Cogn 2011;20:99-108.

39 Burgy M: Ego disturbances in the sense of Kurt Schneider: historical and phenomenological aspects. Psychopathology 2011;44:320-328.

40 Schneider K: Notiz über Ichstörungen und Entfremdungen. Fortschr Neurol Psychiatr 1949; 17:343-347.

41 World Health O: The ICD-10 classification of mental and behavioural disorders : clinical descriptions and diagnostic guidelines. Geneva, World Health Organization, 1992. 42 American Psychiatric A: Diagnostic and Statistical Manual of Mental Disorders, Fifth Edition (DSM-5®). Washington, D.C., American Psychiatric Publishing, 2013.

43 George AL, Bennett A, Belfer Center for S, International A: Case studies and theory development in the social sciences. Cambridge, Mass, MIT Press, 2005.

44 Miller TJ, McGlashan TH, Rosen JL, Cadenhead K, Cannon T, Ventura J, McFarlane W, Perkins DO, Pearlson GD, Woods SW: Prodromal assessment with the structured 
interview for prodromal syndromes and the scale of prodromal symptoms: predictive validity, interrater reliability, and training to reliability. SchizophrBull 2003;29:703-715.

45 Schultze-Lutter F, Addington J, Ruhrmann S, Klosterkotter J: Schizophrenia proneness instrument: Adult version (SPI-A). Roma, Giovanni Fioriti Editore, 2007.

46 First MB: DSM-IV-TR guidebook. Washington, DC, American Psychiatric Pub., 2004.

47 Kimhy D, Jobson-Ahmed L, Ben-David S, Ramadhar L, Malaspina D, Corcoran CM: Cognitive insight in individuals at clinical high risk for psychosis. Early Interv Psychiatry 2014;8:130-137.

48 Uchida T, Matsumoto K, Ito F, Ohmuro N, Miyakoshi T, Ueno T, Matsuoka H: Relationship between cognitive insight and attenuated delusional symptoms in individuals with at-risk mental state. Psychiatry Res 2014;217:20-24.

49 Moller P, Haug E, Raballo A, Parnas J, Melle I: Examination of anomalous selfexperience in first-episode psychosis: interrater reliability. Psychopathology 2011;44:386390.

50 Sass L: Self-disturbance in schizophrenia: hyperreflexivity and diminished selfaffection; in Kircher T, David A (eds): The Self in Neuroscience and Psychiatry. New York, Cambridge University Press, 2003, pp 242-271.

51 Fuchs T: From Self-Disorders to Ego Disorders. Psychopathology 2015;48:324-331.

52 Sierra EM, Berrios EG: The Phenomenological Stability of Depersonalization:

Comparing the Old with the New. The Journal of Nervous and Mental Disease 2001;189:629636.

53 Sass L, Pienkos E, Nelson B, Medford N: Anomalous self-experience in depersonalization and schizophrenia: a comparative investigation. Conscious Cogn 2013;22:430-441.

54 Parnas J, Handest P: Phenomenology of anomalous self-experience in early schizophrenia. ComprPsychiatry 2003;44:121-134.

55 Michal M, Adler J, Wiltink J, Reiner I, Tschan R, Wolfling K, Weimert S, Tuin I, Subic-Wrana C, Beutel ME, Zwerenz R: A case series of 223 patients with depersonalizationderealization syndrome. BMC Psychiatry 2016;16:203.

56 Linscott R, van Os J: An updated and conservative systematic review and metaanalysis of epidemiological evidence on psychotic experiences in children and adults: on the pathway from proneness to persistence to dimensional expression across mental disorders. Cambridge, Cambridge University Press, 2013, 43, pp 1133-1149.

57 Morris SE, Cuthbert BN: Research Domain Criteria: cognitive systems, neural circuits, and dimensions of behavior. Dialogues Clin Neurosci 2012;14:29-37.

58 Linscott RJ, van OJ: Systematic reviews of categorical versus continuum models in psychosis: evidence for discontinuous subpopulations underlying a psychometric continuum. Implications for DSM-V, DSM-VI, and DSM-VII. AnnuRevClinPsychol 2010;6:391-419. 59 Postmes L, Sno HN, Goedhart S, van der Stel J, Heering HD, de Haan L: Schizophrenia as a self-disorder due to perceptual incoherence. Schizophr Res 2014;152:4150 .

60 Gamma F, Goldstein JM, Seidman LJ, Fitzmaurice GM, Tsuang MT, Buka SL: Early intermodal integration in offspring of parents with psychosis. Schizophr Bull 2014;40:9921000.

61 Damasio A: Self comes to mind: Constructing the conscious brain. New York, NY, Pantheon/Random House; US, 2010.

62 Sass L, Byrom G: Phenomenological and neurocognitive perspectives on delusions: A critical overview. World Psychiatry 2015;14:164-173. 
63 Varese F, Smeets F, Drukker M, Lieverse R, Lataster T, Viechtbauer W, Read J, van Os J, Bentall RP: Childhood adversities increase the risk of psychosis: a meta-analysis of patient-control, prospective- and cross-sectional cohort studies. Schizophr Bull 2012;38:661671.

64 Ruby E, Polito S, McMahon K, Gorovitz M, Corcoran C, Malaspina D: Pathways Associating Childhood Trauma to the Neurobiology of Schizophrenia. Frontiers in psychological and behavioral science 2014;3:1-17.

65 Velikonja T, Fisher HL, Mason O, Johnson S: Childhood trauma and schizotypy: a systematic literature review. Psychol Med 2015;45:947-963.

66 Medford N, Baker D, Hunter E, Sierra M, Lawrence E, Phillips ML, David AS: Chronic depersonalization following illicit drug use: a controlled analysis of 40 cases. Addiction 2003;98:1731-1736.

67 Simeon D, Kozin DS, Segal K, Lerch B: Is depersonalization disorder initiated by illicit drug use any different? A survey of 394 adults. J Clin Psychiatry 2009;70:1358-1364. 68 Hurlimann F, Kupferschmid S, Simon AE: Cannabis-induced depersonalization disorder in adolescence. Neuropsychobiology 2012;65:141-146.

69 Medford N, Sierra M, Stringaris A, Giampietro V, Brammer MJ, David AS:

Emotional Experience and Awareness of Self: Functional MRI Studies of Depersonalization Disorder. Front Psychol 2016;7:432.

70 Sierra M, Berrios GE: Depersonalization: neurobiological perspectives. Biol Psychiatry 1998;44:898-908.

71 Stein DJ, Simeon D: Cognitive-affective neuroscience of depersonalization. CNS spectrums 2009;14:467-471.

72 Guralnik O, Giesbrecht T, Knutelska M, Sirroff B, Simeon D: Cognitive functioning in depersonalization disorder. J Nerv Ment Dis 2007;195:983-988.

73 Guralnik O, Schmeidler J, Simeon D: Feeling unreal: cognitive processes in depersonalization. Am J Psychiatry 2000;157:103-109.

74 Simeon D, Hamilton HK: Depersonalization disorder and schizotypal personality disorder; Moskowitz, Andrew [Ed]; Schafer, Ingo [Ed]; Dorahy, Martin J [Ed] (2008) Psychosis, trauma and dissociation: Emerging perspectives on severe psychopathology (pp 209-220) xxi, 358 pp Wiley-Blackwell, 2008

75 Shepherd AM, Laurens KR, Matheson SL, Carr VJ, Green MJ: Systematic metareview and quality assessment of the structural brain alterations in schizophrenia. Neurosci Biobehav Rev 2012;36:1342-1356.

76 Hazlett EA, Goldstein KE, Kolaitis JC: A review of structural MRI and diffusion tensor imaging in schizotypal personality disorder. Current psychiatry reports 2012;14:70-78.

Table 1 EASE-scores and core BSD 1: Diminished Self-Affection

\begin{tabular}{|l|l|l|}
\hline & DPD-case 'Eva" & \multicolumn{1}{c|}{ SPD-case "Robert" } \\
\hline EASE scores & $\begin{array}{l}\text { Total EASE-score: 31 } \\
\text { Domain 1: 12, Domain 2: 11, Domain 3: 5, } \\
\text { Domain 4: 0, Domain 5: 3 }\end{array}$ & $\begin{array}{l}\text { Total EASE-score: 27 } \\
\text { Domain 1: 8, Domain 2: 11, Domain 3: 0, } \\
\text { Domain 4: 1, Domain 5: 7 }\end{array}$ \\
\hline $\begin{array}{l}\text { Quotes on } \\
\text { Diminished } \\
\begin{array}{l}\text { Self-Affection } \\
\text { and } \\
\text { Corresponding } \\
\text { EASE-items }\end{array}\end{array}$ & $\begin{array}{l}\text { f..my 'I', or my soul, feels absent, it doesn't } \\
\text { feel completely as myself... I can suddenly } \\
\text { feel that I don't know who I am... seeing } \\
\text { myself from the outside, not feeling any } \\
\text { connection to myself, it becomes in a way } \\
\text { alien.' } \\
\text { (EASE-item 2.1 Diminished Sense of Basic }\end{array}$ & $\begin{array}{l}\text { I understand that people behave differently in } \\
\text { about it. I feel then that I'm not constant... I } \\
\text { can do it when I'm alone, too, then it's not } \\
\text { making any sense... as if I have passed from } \\
\text { one side of a personality trait to another, and } \\
\text { then I don't understand how I thought about }\end{array}$ \\
\hline
\end{tabular}




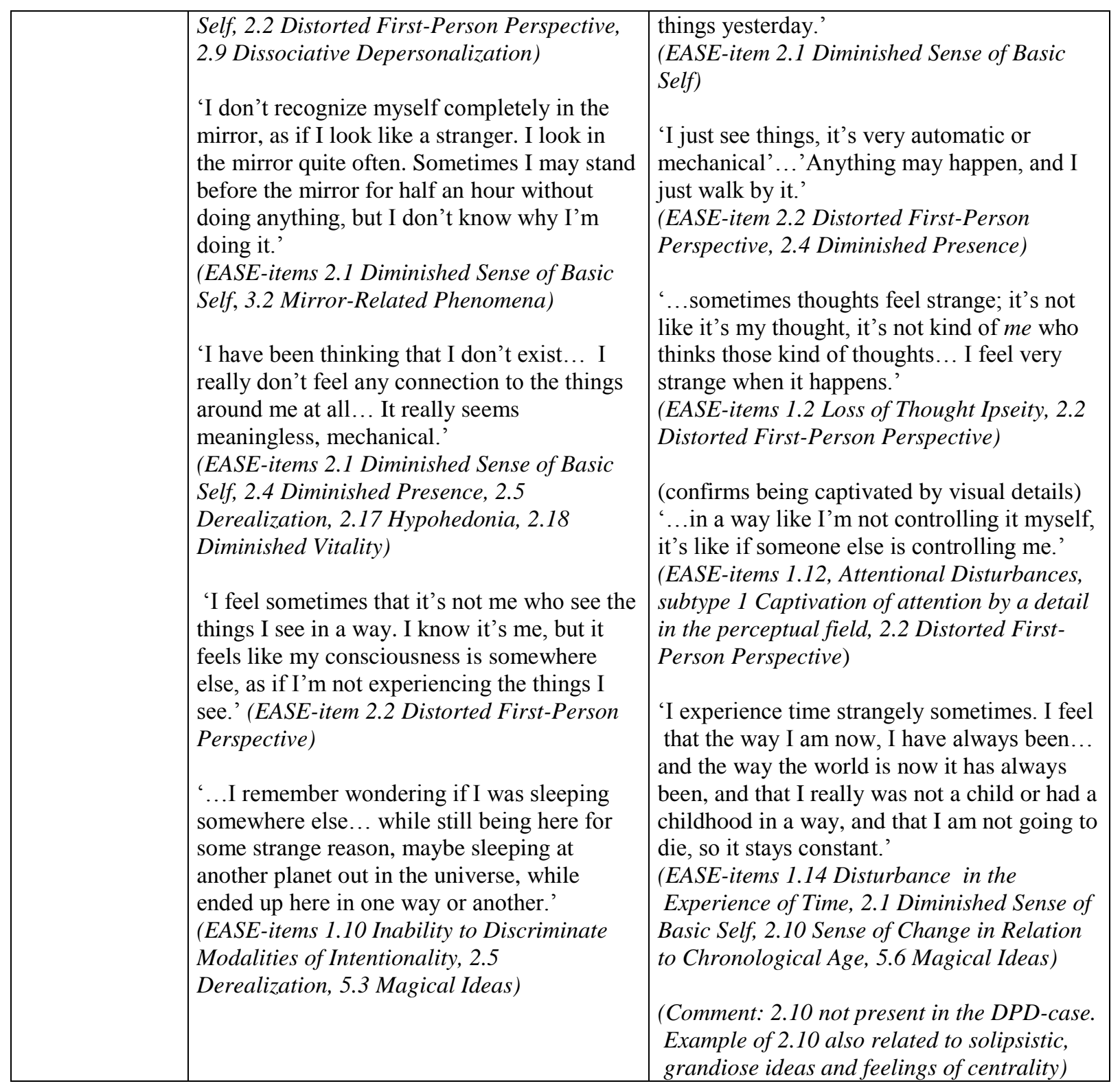

Table 2 EASE-scores and Core BSD 2: Hyperreflexivity

\begin{tabular}{|c|c|c|}
\hline & DPD-case "Eva" & SPD-case "Robert" \\
\hline EASE scores & $\begin{array}{l}\text { Total EASE-score: } 31 \\
\text { Domain 1: } 12 \text {, Domain 2: 11, Domain 3: 5, } \\
\text { Domain 4: 0, Domain 5: } 3\end{array}$ & $\begin{array}{l}\text { Total EASE-score: } 27 \\
\text { Domain 1: 8, Domain 2: 11, Domain 3: 0, } \\
\text { Domain 4: 1, Domain 5: } 7\end{array}$ \\
\hline $\begin{array}{l}\text { Quotes on } \\
\text { Hyperreflexivity } \\
\text { and } \\
\text { Corresponding } \\
\text { EASE-items }\end{array}$ & $\begin{array}{l}\text { 'Earlier, a whole day would pass being } \\
\text { involved in a lot of activities: being with } \\
\text { friends, at school, going to a store, and } \\
\text { everything had a flow to it. If I thought } \\
\text { about something, it was kind of natural..., } \\
\text { but now nothing is flowing naturally. I think } \\
\text { about everything quite often.' } \\
\text { (EASE-items } 1.14 \text { Disturbance in } \\
\text { Experience of Time, } \\
\text { 2.2 Distorted First-Person Perspective, } \\
\text { 2.6 Hyperreflectivity) }\end{array}$ & $\begin{array}{l}\text { 'Some days everything feels unreal... as if I } \\
\text { was a fly on the wall sitting and observing } \\
\text { what I am doing: cooking, going to bed, and } \\
\text { suddenly the day has passed' } \\
\text { (EASE-items } 2.2 \text { Distorted First-Person } \\
\text { Perspective, } 2.4 \text { Diminished Presence, } 2.8 \\
\text { Dissociative Depersonalization) } \\
\text { 'I think a lot about the body language (of } \\
\text { friends), compared with what they say and } \\
\text { do, and it makes me confused. I feel that I } \\
\text { should know this person and his body }\end{array}$ \\
\hline
\end{tabular}




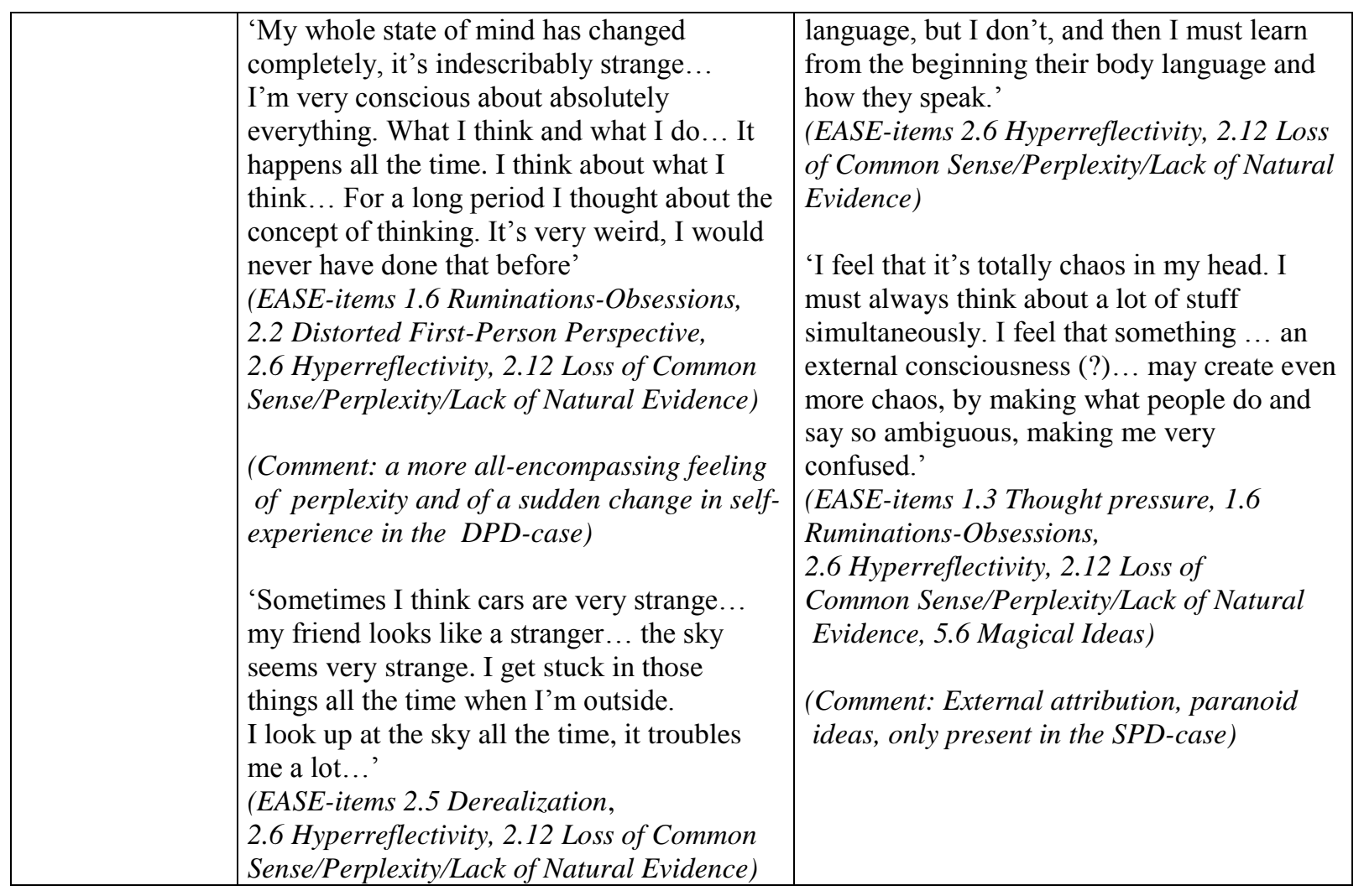

Table 3 Depersonalization syndrome

\begin{tabular}{|c|c|c|}
\hline & \multicolumn{2}{|c|}{$\begin{array}{l}\text { Quotes on the Depersonalization Syndrome and Corresponding } \\
\text { EASE-items }\end{array}$} \\
\hline & DPD-case "Eva" & SPD-case "Robert" \\
\hline $\begin{array}{l}\text { Symptom group } 1 \\
\text { Feelings of } \\
\text { disembodiment }\end{array}$ & $\begin{array}{l}\text { 'I get this strange feeling that I'm only } \\
\text { walking around like a piece of meat, and } \\
\text { as if my soul is not connected to... } \\
\text { (pointing to the body), that my soul is } \\
\text { somewhere else.' } \\
\text { (EASE-items 2.1 Diminished Sense of } \\
\text { Basic Self, 2.2 Distorted First-Person } \\
\text { Perspective, } 3.4 \text { Psychophysical Misfit and } \\
\text { Psychophysical Split, 3.6 Spatialization of } \\
\text { Bodily Experiences) } \\
\text { (Comment: Items from domain } 3 \text { only } \\
\text { present in the DPD-case }\end{array}$ & $\begin{array}{l}\text { 'Some days everything feels unreal... as if } \\
\text { I was a fly on the wall sitting and } \\
\text { observing what I am doing: cooking, going } \\
\text { to bed and suddenly the day has passed.' } \\
\text { (EASE-items } 2.2 \text { Distorted First-Person } \\
\text { Perspective, } 2.4 \text { Diminished presence, } 2.8 \\
\text { Dissociative Depersonalization) } \\
\text { (Comment: hyperreflexivity and lack of } \\
\text { agency feelings, but denies the presence of } \\
\text { items from domain 3) }\end{array}$ \\
\hline $\begin{array}{l}\text { Symptom group } 2 \\
\text { Emotional numbing }\end{array}$ & $\begin{array}{l}\text { 'I never experience joy or sadness, it's a } \\
\text { complete lack of feeling ... It's with } \\
\text { respect to everything, like food. I } \\
\text { remember earlier: let's say I came home } \\
\text { from training... then I drank a coke, and it } \\
\text { was very satisfying. I never have that } \\
\text { feeling anymore.' } \\
\text { (EASE-items 2.4 Diminished Presence, } \\
\text { 2.17 Hypohedonia, 2.18 Diminished } \\
\text { Vitality) }\end{array}$ & $\begin{array}{l}\text { '...I may say "I am sorry", but I feel } \\
\text { nothing.' } \\
\text { 'My girlfriend has called me egocentric } \\
\text { and robot-like... I have been wondering if } \\
\text { I may have the qualities of a robot, but not } \\
\text { that I am a robot.' } \\
\text { (EASE-item 2.1 Diminished sense of basic } \\
\text { self, 2.4 Diminished presence, } 2.17 \\
\text { Hypohedonia, } 2.18 \text { Diminished Vitality ) }\end{array}$ \\
\hline $\begin{array}{l}\text { Symptom group } 3 \\
\text { Anomalous }\end{array}$ & $\begin{array}{l}\text { 'Some times when playing basketball with } \\
\text { friends, it happens quite often that I ask }\end{array}$ & $\begin{array}{l}\text { 'I wonder if something really happened or } \\
\text { if I dreamt it, or if I thought about it in }\end{array}$ \\
\hline
\end{tabular}




\begin{tabular}{|c|c|c|}
\hline subjective recall & $\begin{array}{l}\text { them: "Is this something I have dreamt... } \\
\text { or did it really happen?" I have to be } \\
\text { reassured by others.' } \\
\text { (EASE-item } 1.10 \text { Inability to Discriminate } \\
\text { Modalities of Intentionality }\end{array}$ & $\begin{array}{l}\text { such a strong way that I, in the last years, } \\
\text { have begun to believe it.' } \\
\text { 'Thinking about (my) childhood, I feel } \\
\text { those thoughts are put into my head.' } \\
\text { (EASE-items } 1.2 \text { Loss of Thought Ipseity, } \\
\text { 1.10 Inability to Discriminate Modalities } \\
\text { of Intentionality, } 5.6 \text { Magical Ideas) } \\
\text { (Comment: External, paranoid } \\
\text { attribution, only present in the SPD-case) }\end{array}$ \\
\hline $\begin{array}{l}\text { Symptom group } 4 \\
\text { Derealization }\end{array}$ & $\begin{array}{l}\text { '...they (surroundings) really seem } \\
\text { meaningless, mechanical.' } \\
\text { '... it felt like I was in a movie, it looked } \\
\text { like... a classic movie, Hollywood-like.' } \\
\text { 'I felt that something truly was wrong, that } \\
\text { everything could fall apart.' } \\
\text { (EASE-items } 2.5 \text { Derealization, } 2.14 \\
\text { Ontological Anxiety, } 5.5 \text { 'As-if' Feeling } \\
\text { that the Experienced World Is Not Truly } \\
\text { Real, Existing, as if It Was Only Somehow } \\
\text { Apparent, Illusory or Deceptive) }\end{array}$ & $\begin{array}{l}\text { 'Yes, I feel that it's something wrong in } \\
\text { me, but also in the world around me... I } \\
\text { can see it, or realize it... I feel that } \\
\text { everything is fake, feigned or planned, that } \\
\text { it is too systematized, making me think } \\
\text { that it cannot be a coincident.' } \\
\text { (EASE-items } 2.5 \text { Derealization, } 5.5 \text { 'As-if' } \\
\text { Feeling that the Experienced World Is Not } \\
\text { Truly Real, Existing, as if It Was Only } \\
\text { Somehow Apparent, Illusory or Deceptive, } \\
\text { 5.6 Magical Ideas) } \\
\text { (Comment: External, paranoid } \\
\text { attributions, only present in the SPD-case) }\end{array}$ \\
\hline
\end{tabular}

